of ACR and were randomly collected in clinical remission state (SLEDAI 1.1 \pm 1.9 ).

Results By comparison of B cell subsets between SLE and HC, CD38 was dominantly expressed by SLE patients (SLE 74.2\% $\pm 12.9 \%$ vs HC $64.2 \% \pm 12.2 \%$; p(MWU) $=0.018)$. Furthermore, SLE-patients showed an increase in CD19 + IgD CD27 + CD 38 high plasmablasts (SLE $2.1 \% \pm 3.4 \%$ vs HC $0,4 \%$ $\pm 0.4 \%, \mathrm{p}(\mathrm{MWU})<0.001)$. Moreover, SLE-plasmablasts showed decreased CD73 expression as compared to HC (SLE 2.1\% $\pm 1.9 \%$ vs $\mathrm{HC} 3.5 \% \pm 2.2 \%$; $(\mathrm{MWU})=0.034)$. SLE-B cells revealed a trend towards an augmented CD38highCD138 +plasmacell fraction (SLE $0.40 \% \pm 0.5 \%$ vs HC $0.08 \%$ $\pm 0.07 \%$; $\mathrm{p}=0.07$ ), without any difference in CD73 expression. On the other hand, exhausted-memory B cell fraction (CD19 + IgD CD27-CD21-CD138-), showed an increased CD73 expression in SLE (SLE $13.7 \% \pm 9.2 \%$ vs HC $6.2 \%$ $\pm 5.4 \% ; \mathrm{p}=0.004)$.

Conclusion Our study confirms CD38 + plasmablasts as being increased in peripheral blood from SLE patients as compared to HC. Furthermore, the data reveal a deficiency for CD73 on SLE plasmablasts, which suggests a decreased regulatory capacity of SLE plasmablasts as compared to HC, supporting the notion of a reduced regulatory $\mathrm{B}$ cell pool in SLE. On the other hand, the enlarged CD73 + exhausted memory pool in SLE could point to an accelerated flow of CD73 + regulatory B cells into an exhausted B cell fraction. These findings support the hypothesis of a persistent regulatory B cell defect even in a state of SLE remission.

\section{PS5:90 ENHANCED IL-7 RECEPTOR SIGNALLING IN SLE PROMOTES T-HELPER CELL PROLIFERATION THROUGH UPREGULATION OF MICRORNA-182 AND DOWNREGULATION OF FOX01}

${ }^{1} \mathrm{~T}$ Alexander, ${ }^{2} \mathrm{C}$ Haftmann, ${ }^{2} \mathrm{R}$ Riedel, ${ }^{1} \mathrm{~L}$ Templin, ${ }^{1} \mathrm{G}$ Burmester, ${ }^{2} \mathrm{~A}$ Radbruch, ${ }^{1} \mathrm{~F}$ Hiepe ${ }^{2} \mathrm{~F}$ Mashreghi. ${ }^{1}$ Charité University Medicine, Department of Rheumatology, Berlin, Germany; ${ }^{2}$ Deutsches Rheuma-Forschungszentrum, Berlin, Germany

\subsection{6/lupus-2018-abstract.135}

Background Recent reports have shown dysregulated microRNAs (miRNAs) in murine models of lupus, among them increased expression of microRNA-182 (miRNA-182), which has been demonstrated to target the transcription factor FOXO1 in activated murine CD4 $+\mathrm{T}$ cells, leading to spontaneous $\mathrm{T}$ cell activation and clonal expansion. Here we aimed to investigate the expression of miR-182 and FOXO1 in $\mathrm{T}$ cells from human SLE patients.

Methods Expression levels of miR-182 were analysed with RTPCR in purified peripheral blood CD4 $+\mathrm{T}$ cells from 9 patients with SLE and age/sex-matched healthy controls (HC). Multicolor flow cytometry was performed to analyse CD4 + T cell expression for FOXO1, Ki-67, Foxp3, the interleukin-7 receptor- $\alpha$ (CD127) and phosphorylated STAT-5a (pSTAT5). Analysis of serum IL-7 levels was performed with ELISA in 27 SLE patients and HC. Induction of miR-182 was assessed in vitro after polyclonal $\mathrm{T}$ cell stimulation in the presence of IL-7, and inhibition of T cell proliferation investigated using mir-182 antagomirs.

Results MiRNA-182 was significantly upregulated in CD4 +T cells from SLE patients compared to $\mathrm{HC}$, while the FOXO1 expression was significantly decreased. The percentage of proliferating Ki-67 + conventional Foxp3- CD4 + T cells (Tcons) was significantly higher in SLE compared to HC $(3.85 \%$ vs
$1.58 \%, \mathrm{p}<0.001)$ and their basal pSTAT5 levels significantly enhanced, suggesting a recent stimulation with common gamma chain $(\gamma c)$-signalling cytokines. SLE Tcons displayed decreased expression levels for the FOXO1 target gene CD127 (MFI 2021 vs 2553, p=0.049) and serum IL-7 levels were significantly higher in SLE compared to HC $(17.0 \mathrm{pg} / \mathrm{ml}$ vs $10.2 \mathrm{pg} / \mathrm{ml}, \mathrm{p}=0.001)$. In vitro, miR-182 could be induced by IL-7, and specific inhibition of miR-182 inhibited $\mathrm{T}$ cell proliferation and survival.

Conclusion Our data suggest that enhanced IL 7R/STAT5 signalling mediates the induction of miR 182 expression, which promotes the proliferation of conventional Foxp3- T cells SLE. Collectively, our data provide new insights in the pathophysiology of $\mathrm{T}$ cell hyperactivity in SLE and identifies miR-182 as a candidate target for future therapeutic approaches.

\section{PS5:91 INCREASED EXPRESSION OF TLR7 IN PLASMACYTOID DENDRITIC CELLS DRIVES TYPE I IFN MEDIATED IMMUNOPATHOLOGY IN SLE AND APS}

${ }^{1} \mathrm{LL}$ van den Hoogen, ${ }^{1} \mathrm{~A}$ Pandit, ${ }^{1} \mathrm{G}$ Palla, ${ }^{1} \mathrm{M}$ Rossato, ${ }^{2} \mathrm{RDE}$ Fritsch-Stork, ${ }^{1} \mathrm{JAG}$ van Roon, ${ }^{1}$ TR Radstake. 'Laboratory of Translational Immunology/Rheumatology and Clinical Immunology, University Medical Centre Utrecht, The Netherlands; ${ }^{2} 1$ st Medical Department, Hanusch Hospital of WGKK, Sigmund Freud University, Ludwig Boltzmann Institute of Osteology, Vienna, Austria

\subsection{6/lupus-2018-abstract.136}

Background/purpose Dendritic cells (DC) are key cells in the pathogenesis of autoimmune diseases by potently activating $\mathrm{T}$ cells. Systemic lupus erythematosus (SLE) and antiphospholipid syndrome (APS) are characterised by an IFN signature, caused by elevated levels of IFNo. Plasmacytoid DC (pDC) are held responsible for the increased levels of IFN $\alpha$ in SLE and APS. The molecular mechanisms underlying the increased activation of pDC in SLE and APS are unknown. Using RNA sequencing (RNAseq) and further in vitro validation experiments on plasmacytoid and myeloid DC (mDC) obtained from patients with SLE and APS we assessed the causes and consequences of increased type I IFN signalling on the dysregulation of both pDC and $\mathrm{mDC}$ in patients with SLE and APS.

Methods RNAseq was performed on $\mathrm{pDC}$ and $\mathrm{mDC}$ isolated from peripheral blood of patients with SLE, SLE +APS and primary APS (PAPS) and healthy controls $(n=54)$. Weighted gene correlation network analysis (WGCNA) was used to identify pDC- and mDC-specific gene modules and to stratify patients into those with (IFN-high) or without (IFN-low) an IFN signature. The response of $\mathrm{pDC}$ and $\mathrm{mDC}$ (in co-culture with T-cells) to IFN $\alpha$ and TLR agonists were analysed by RTqPCR and flow cytometry to functionally validate RNAseq data.

Results WGCNA identified IFN modules in pDC and mDC that perfectly stratified patients from HC. Comparing the IFN modules of $\mathrm{pDC}$ and $\mathrm{mDC}$ revealed cell specific alterations related to the IFN signature in $\mathrm{pDC} / \mathrm{mDC}$. Increased expression of TLR7 and its downstream intermediates was confined to IFN-high patients in pDC. In contrast, genes involved in the activation of T-cells were related to the IFN module in $\mathrm{mDC}$. Both $\mathrm{pDC}$ and $\mathrm{mDC}$ showed increased expression of BAFF. In vitro, IFNo upregulated TLR7 in pDC and augmented TLR7-mediated IFN $\alpha$ production. In contrast to pDC, IFN $\alpha$ primed $\mathrm{mDC}$ for enhanced T-cell proliferation via the upregulation of co-stimulatory molecules. Furthermore, in SLE/ 